\title{
Two-Stage Method for Optimal Operation of a Distributed Energy System
}

\author{
Jie Xue, Jue Zhou and Yaobin Chen \\ School of Electrical and Computer Engineering \\ Indiana University Purdue University Indianapolis \\ Indianapolis, Indian USA \\ jiexue@iupui.edu
}

\begin{abstract}
In this paper, a gas turbine-based distributed energy system (DES) model is developed for the design of operation planning. An operation mode aimed to optimize the operation of this DES is proposed. A multi-objective cost function considering the total system efficiency and operational cost is formulated for the optimal design of DES operation and control. A two-stage approach combining the particle swarm algorithm (PSO) with the sequential quadratic programming (SQP) method is employed to solve the nonlinear programming problem. Optimal operation strategies for the DES are investigated using the proposed twostage method under three different demand loads in terms of weather conditions. The simulation results are compared with those using traditional rule-based operation methods. It is found that under the proposed operation mode, the DES is capable of achieving an improved performance in terms of thermal efficiency and operational cost.
\end{abstract}

Index Terms-Distributed generation, Optimization, Operational cost, System efficiency, Two-stage method

\section{INTRODUCTION}

Distributed generation (DG) can be defined as electric power generation within distribution networks or on the customer site of the network [1]. With the widespread application of combined heating and cooling (CHP) and combined cooling, heating and power (CCHP), the generated energy includes not only electricity, but heating and cooling as well. Compared to large, centralized conventional power plant, distributed generation has several competitive benefits. It is close to the user-end which could lead to the reduction of both electricity transmission loss and thermal transport loss. Moreover, DG is capable of applying renewable energy resources (e.g. solar, wind and biomass) to reduce carbon footprint. In addition, DG is able to meet the local demands in extreme conditions when the connection with the main grid is unavailable, so that the energy supply security could be enhanced. Due to the advantages of DG, it is well acknowledged that DG would play an important role in future's energy supply.

In spite of its relatively small scale and low voltage, distributed energy system (DES) is a complicated energy system, in terms of varied load demands and the corresponding multienergy generation. In order to achieve the load demands while maximizing the economic and energy saving benefits, the operation management of DES is crucial. Among the various aspects influencing the DES operation, the operation strategy is an important factor determining the performance of DES.
Much research has been carried out and a wide range of mathematical models have been developed, aiming to optimize DES operations [1-4]. The most commonly used operation strategy for DES is rule-based methods, which control the system by either following the thermal loads or following the electrical loads. However, these two empirical rule-based methods only focus on meeting the load demands. Therefore, it is difficult to take into account of other important aspects, such as energy saving, operational cost, environmental impact. For a system for multiple objectives, rule-based methods normally may not provide an optimal solution. Instead, optimization algorithm would be an alternative way to pursue these goals. For a DES model based on component models, the operation optimization problems can be mathematically considered as a linear programming problem or nonlinear programming problem. A variety of methods has been applied to achieve the optimal operation strategy, such as the Simplex method, Lagrangian relaxation method, quadratic programming method, etc. [2].

When evaluating the performance and benefits of DES, it is required to identify performance measures first. According to the different goals of optimization, different types of performance measures have been proposed [3-8]. Usually, three aspects are taken into consideration, which are system efficiency, operational cost, and environment impact, respectively.

Primary energy saving (PES) is used to assess the energy consumption difference between DES and separate generation (SG) conventional systems. It is also called 'TPES' when using for trigeneration [4]. Primary energy savings ratio (PESR) could be applied to evaluate the primary energy saving achieved by DES with respect to the reference conventional system. Energy utilization factor (EUF) based on the first law of thermodynamics is defined as the combined energy of the flows produced by the cogeneration system and used to meet the energy demands (electricity, hot water, steam and chilled water) divided by the energy consumption of engine [5]. Carbon dioxide emissions and carbon dioxide emission ratio are defined to evaluate the environment impact. The amount of emission is estimated using the fuel conversion factors and electricity factors [7, 8].

In this paper, an integrated DES system model is developed based on detailed component models. Both the operational cost and exergy efficiency are considered together as a multi- 
objective for operation optimization. The optimization problem is formulated as a nonlinear programming problem with 672 variables, 288 equality constraints and 576 inequality constraints. A two-stage method consisting of a particle swarm algorithm (PSO) and the sequential quadratic programming (SQP) is employed to solve the problem. Three cases with varied loads at three different seasons have been investigated in detail. It is found that the performance of DES under the proposed operation mode is better than those using traditional rule-based operation methods, in terms of the operational cost and exergy efficiency.

\section{SYSTEM CONFIGURATION AND COMPONENT MODELS}

Fig. 1 shows the configuration of a gas turbine based DES. This DES consists of a $350 \mathrm{KW}$ micro-gas turbine generator, a $600 \mathrm{KW}$ absorption chiller, a $100 \mathrm{KW}$ electricity chiller and a $600 \mathrm{KW}$ heat exchanger. Natural gas is used as the fuel being burned in the gas turbine to drive a synchronous generator. Waste heat from the gas turbine is distributed into heat exchanger and absorption chiller to generate hot water and cold water, respectively. Electric chiller will be turned on when the cooling output of absorption chiller is not sufficient. Twodirectional electrical flow with the power grid is allowed, so the excess electricity may be sold back to the grid depending on the energy policy.

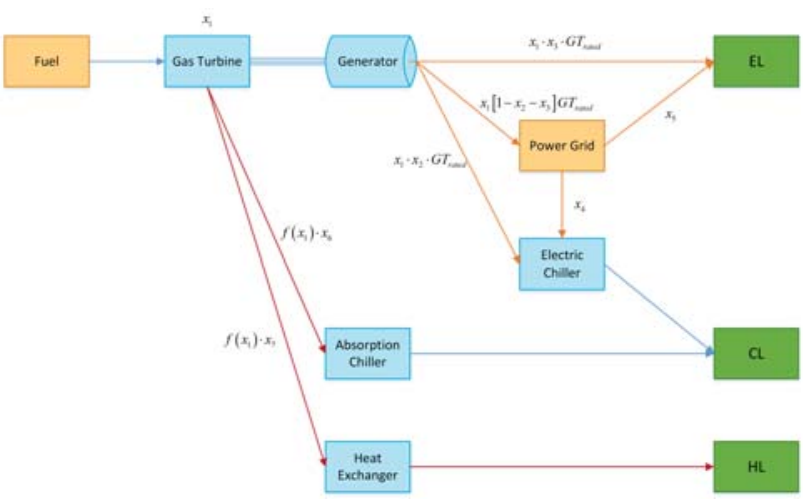

Fig. 1: The configuration of DES.

\section{A. Gas Turbine Model}

According to Wang [9] and Zhang [10], the main outputs of a micro-gas turbine model include exhaust gas temperature $T_{4}$, mass flow rate of exhaust gas $G_{g}$, and mass flow rate of fuel $G_{f}$, which can be represented as a function of output power $P$ as follows:

$$
\begin{aligned}
& T_{4}(P)=a_{1} P^{3}+a_{2} P^{2}+a_{3} P+a_{4} \\
& G_{g}(P)=b_{1} P^{2}+b_{2} P+b_{3} \\
& G_{f}(P)=c_{1} P^{2}+c_{2} P+c_{3}
\end{aligned}
$$

where $a, b$ and $c$ are coefficients.

\section{B. Heat Exchanger Model}

Using the $\varepsilon$-NTU method, the heat exchange is considered as a function of exhaust gas mass flow $G$ and temperature $T$ [18], as given in (4). For the heat exchanger being considered in this work, the relations among the exhaust gas mass flow rate, its temperature, and the exchange heat amount are presented in Fig. 2, and can be expressed as a lookup table. A two-dimensional interpolation method is applied in the DES operation optimization.

$$
Q_{H X}=H X(T, G)
$$

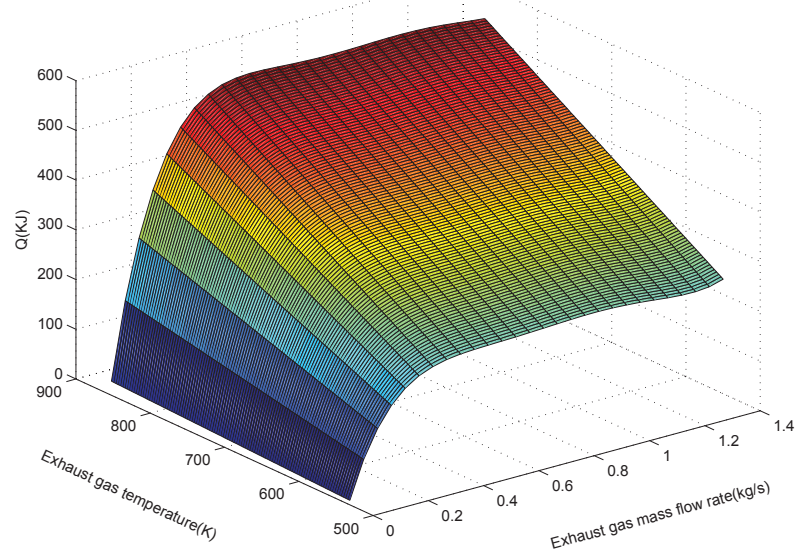

Fig. 2: Heat exchanger performance varied with the exhaust gas mass flow and temperature.

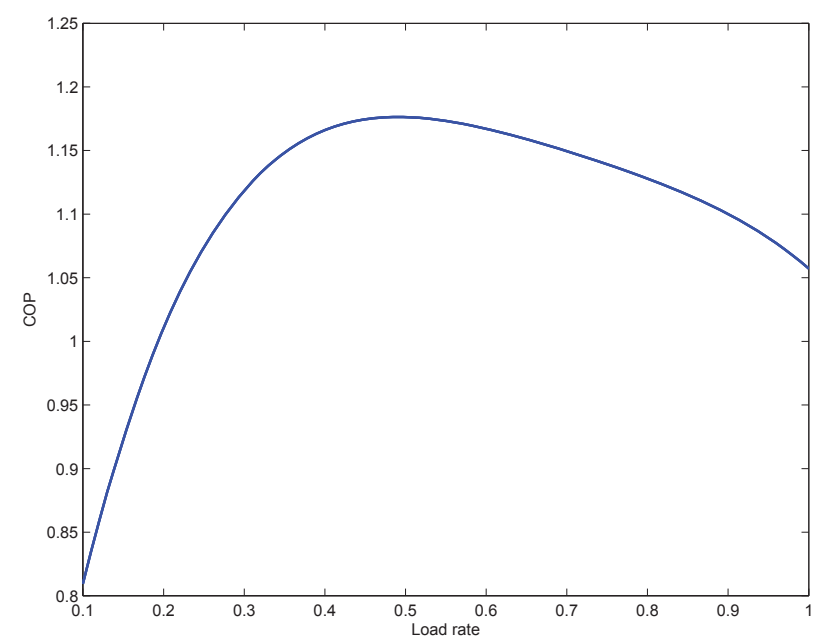

Fig. 3: The relation between COP and load rate for Absorption chiller.

\section{Absorption Chiller Model}

Based on the heat balance and mass balance equations developed by Borg and Kelly [11], the coefficient of performance of absorption chiller can be considered as a function of waste 
heat temperature, as shown in Fig. 3. The absorption chiller capacity is also treated as a function of exhaust gas mass flow and temperature, as follows:

$$
\begin{aligned}
& Q_{A C}=A C(T, G) \\
& C O P_{A C}=C O P(T)
\end{aligned}
$$

\section{Electrical Chiller Model}

For electrical chiller, its efficiency changes little and can be considered as a constant value. Usually, the coefficient is between 3 and 6 . In this study, $C O P_{E C}$ is chosen to be a constant value of 4 . The performance of electrical chiller can be expressed as:

$$
Q_{E C}=E C(P)=C O P_{E C} \cdot P
$$

\section{PROBLEM Formulation}

DES is a complex system including power generation, heat conversion, heat transfer, power transfer, grid interface with the dynamic loads requirement. In this paper, a multi-objective cost function is used to optimize the operational cost and the total system efficiency. The reason the environment impact is not considered in the objective function is because it is correlated with the system efficiency. Normally, the high system efficiency corresponds to the low environmental impact, and vice versa.

\section{A. Cost Function}

The operational cost $f_{\text {cost }}$ is determined by the fuel consumption, the electricity purchased from the grid or sold back to the grid.

$$
f_{\text {cost }}(x)=c_{\text {elec }}^{p} W^{p}(x)+c_{\text {gas }} m_{\text {fuel }}(x)-c_{\text {elec }}^{s} W^{s}(x)
$$

where $c_{\text {elec }}^{p}$ is the electricity purchase price, $c_{\text {elec }}^{s}$ is the electricity selling price, and $c_{\text {gas }}$ is the natural gas price. The total system exergy efficiency $f_{E U F}$ can be calculated based on the fuel exergy, the thermal product, and electricity.

$$
f_{E U F}(x)=\frac{W(x)+E_{h t}(x)+E_{c w}(x)}{m_{\text {fuel }}(x) L H V_{\text {fuel }}}
$$

where $W$ is the power generation, $E_{h t}$ is the exergy for heating demand, $E_{c w}$ is the exergy for cooling demand, $m_{f u e l}$ is the fuel consumption, $L H V$ is the lower heating value of fuel, chosen to be $43100 \mathrm{KJ} / \mathrm{Kg}$.

For the DES in Fig. 1, the decision variables are defined as: $x_{i}$ (1) for GT power output, $x_{i}(2)$ for GT power ratio to EC, $x_{i}(3)$ for GT power ratio to EL, $x_{i}$ (4) for grid power ratio to $\mathrm{EC}, x_{i}(5)$ for grid power ratio to EL, $x_{i}(6)$ for exhaust gas distribution to absorption chiller, and $x_{i}(7)$ for exhaust gas distribution to heat exchange, where $i=1,2, \ldots, 96$ for a 24 hour operation cycle, sampled every 15 minutes. All variables are scaled between 0 and 1 .

For the operational cost, it is preferred to be as small as possible, while for the efficiency, the higher the better. However, there exists a conflict between cost and efficiency. Hence, the optimization algorithm is applied to find out the best tradeoff between the two. In order to balance the multiobjective function, weighting factors are added. In the process of normalization, cost and EUF are compared with extreme values respectively, to achieve a convergent numerical solution.

$$
\min _{x} J(x)=w_{1} \cdot \frac{\sum f_{\text {cost }}(x)}{\sum f_{\text {cost }}^{o}}+w_{2} \cdot \frac{\sum f_{E U F}^{o}}{\sum f_{E U F}(x)}
$$

where $w_{1}, w_{2}$ are weighting factors with $w_{1}+w_{2}=1, f_{\text {cost }}^{o}$ is the cost only condition, $f_{E U F}^{o}$ is the efficiency only condition. The solutions of these two extreme conditions can be achieved through rule-based algorithm.

\section{B. Constraints}

The equality and inequality constraints are listed bellow:

(1)The generated electricity, heating, and cooling have to meet the load demands.

$$
\begin{aligned}
& x_{i}(1) \cdot x_{i}(3) \cdot P_{G T}^{\text {rated }}+x_{i}(5) \cdot E L(i)=E L(i) \\
& A C\left(x_{i}(1), x_{i}(2), x_{i}(4)\right) C O P\left(x_{i}(1)\right)+ \\
& E C\left(x_{i}(1), x_{i}(6)\right)=C L(i) \\
& H X\left(x_{i}(1), x_{i}(7)\right)=H L(i)
\end{aligned}
$$

(2) All units should be operated within upper and lower bounds.

The gas turbine power generation:

$$
P_{l b} \leq P_{G T} \leq P_{u b}
$$

The electrical chiller:

$$
x_{i}(1) \cdot x_{i}(2) \cdot P_{G T}^{\text {rated }}+x_{i}(4) \cdot P_{E C}^{\text {rated }} \leq P_{E C}^{\text {rated }}
$$

The absorption chiller:

$$
A C_{l b} \leq A C\left(x_{i}(1), x_{i}(2), x_{i}(4)\right) \leq A C_{u b}
$$

The heat exchanger:

$$
H X_{l b} \leq H X\left(x_{i}(1), x_{i}(7)\right) \leq H X_{u b}
$$

(3) The power output rising and falling rates constraints

$$
\frac{\left|P_{i+1}-P_{i}\right|}{\Delta i} \leq r_{i}
$$

$\Delta i$ is the time interval between time $i+1$ and $i . r_{i}$ is the maximum rising and falling rates. The maximum rising and falling rates are usually considered as the same value, which can be chosen between 1-5\% per minute.

(4) Energy distribution constraints include:

$$
\begin{aligned}
& x_{i}(2)+x_{i}(3) \leq 1 \\
& x_{i}(6)+x_{i}(7) \leq 1
\end{aligned}
$$

In summary, the optimization problem can be formulated as a standard nonlinear programming problem below:

$$
\begin{aligned}
& \min _{x} J(x) \\
& \text { s.t. } \quad h(x)=0 \\
& \quad g(x) \leq 0 \\
& 0 \leq x_{i}(j) \leq 1 \\
& i=1,2, \ldots, 96, j=1,2, \ldots, 7
\end{aligned}
$$

where $J(x)$ is defined in (10), $h(x)$ consists of (11), (12) and (13), and $g(x)$ is given by (15) to (20). 


\section{Control Strategy}

DES can be controlled by several possible operation modes. In practical operation, the most commonly used operation strategies are rule-base strategies. However, the rule-based strategies are incapable to evaluate the system's performance sufficiently. Hence, an optimal operation mode is proposed in this paper, aiming to increase the system efficiency while decrease the operational cost. For DES without thermal storage, the offline planning problem can be formulated as the nonlinear programming (NLP) problem. Various algorithms can be used to solve this kind of problem. Facci [12] chose dynamic programming method to optimize the CHP system's operation strategy for economic analysis. Chandan [17] used an interiorpoint algorithm, while Rong [13] used Lagrangian relaxation to solve a similar problem. In the current study, considering the problem size and the computational complexity, a twostage approach combining PSO and SQP is employed to solve the NLP problem.

\section{A. The Two-Stage Approach}

PSO is an effective global optimization algorithm for both constrained and unconstrained problems. The PSO algorithm searches in parallel using a group of particles, each particle is a potential solution. For heuristic algorithm like PSO, there are ways to deal with general constrained nonlinear problems [14]. Penalty function is applied to deal with both constraints [20]. In order to reduce the computation time, equality constraints are converted into inequality constraints with a small range. However, there is no guarantee of the global optimum with nonlinear constraints, especially with high dimensional nonlinear inequality constraints [20]. Therefore, the results of PSO are used as the initial guess for SQP to obtain a convergent solution.

The SQP method is a commonly used nonlinear programming method to deal with nonlinear constrained optimization problems. For each iteration, a quadratic programming (QP) subproblem is solved to obtain the search direction for updating the control variables [15]. SQP algorithm is extremely sensitive to the initial guess, especially for large scale problems. A bad initial guess would result in the local optimal, immature results or high computation cost. In this paper, results from PSO is applied as the initial guess to reduce the computational cost.

\section{B. Rule-Based Approach}

Generally, the rule-based algorithms used in DES are following the electrical load method (FEL), following the thermal load method (FTL) and the combination of the two methods (CET).

FEL method means that the electric demand decides the power output of the power generation unit (PGU) if the requirement is below the PGU's rated power. Otherwise, the system should purchase extra electricity from the grid to meet the requirement. Meanwhile, the waste heat is used for cooling and heating consumption.
FTL method means that the PGU generates substantial heat to meet the cooling and heating demands. At the same time, if the PGU does not generate adequate electricity to balance the electric load, the shortfall comes from the grid. And electricity can be sold back to the grid if there is surplus.

Simple operation strategies like FEL and FTL consider load demands only. There might exist better feasible solution when the operational cost and emission aspects are taken into consideration. In order to improve the performance, some researchers use a hybrid electric-thermal load operation mode to reduce both operational and environmental costs $[15,16]$.

In this work, the rule-based algorithms are also applied on the DES model for comparison purposes. Two extreme conditions based on the rule-based approach are simulated, considering either the operational cost or the thermal efficiency. For operational cost only condition, because electricity sold back to the grid is allowed and the electricity selling price is always higher than the purchase price, the power generation unit would always work on the rated power to generate as much electricity as possible. For efficiency only condition, in order to reduce the waste of heat, the system may operate under the FTL method, and therefore the system efficiency is able to achieve its maximum.

\section{Simulation and Discussion}

\section{A. Load Models}

Load models of heating, cooling, and electricity are established by using EnergyPLus. The climate is classified into three types: summer, winter, and transition season between the two. The modeling results of demand loads at three different seasons are shown in Fig. 4. The red line indicates the heating load, blue for the cooling load and green for the electricity load.

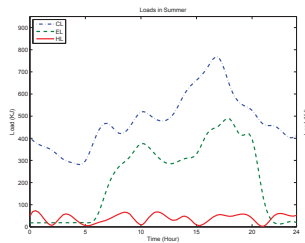

(a) Summer loads

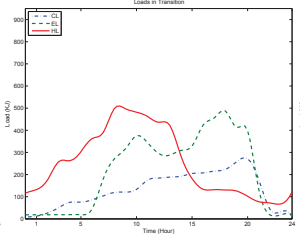

(b) Transition loads

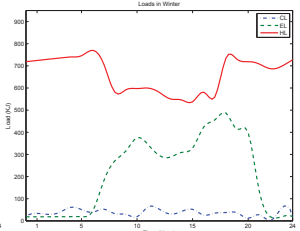

(c) Winter loads
Fig. 4: Demand loads under different seasons.

\section{B. Simulation Results}

Fig. 5 shows the modeling results of EUF at the load in summer. Four cases are compared, which are the cost only case, EUF only case, CET case, and optimized mode case, respectively. It is undoubted that the EUF only case has the highest effectiveness among four cases. The optimized mode case can achieve the efficiency as high as the EUF only case, during several time periods. However, it is noted that the CET case occasionally has a better performance than the optimized mode case. The cost only case always has the lowest efficiency compared to other three cases. In terms of the average EUF for 
a period of 24-hour operation, it can be seen that the efficiency of optimized mode case is approximately $2 \%$ lower than the EUF only case, but is $3.5 \%$ higher than the CET case.

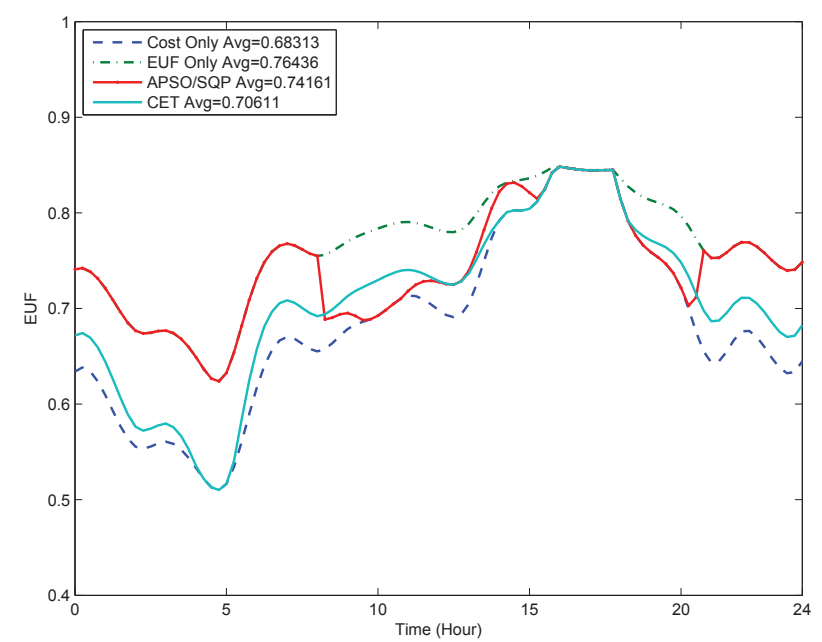

Fig. 5: EUF comparison in summer.

In terms of the operational cost, it is found that the trajectory of optimized mode case is always between the two extreme cases (i.e. cost only case and EUF only case), as shown in Fig. 6. Both Figs. 5 and 6 suggest that the optimized solution focuses more on the efficiency at night when both thermal load and electricity load are low. While during the daytime when all loads are high, the operational cost turns to be more important. As a result, the average efficiency of optimized mode case is better than the cost only solution.

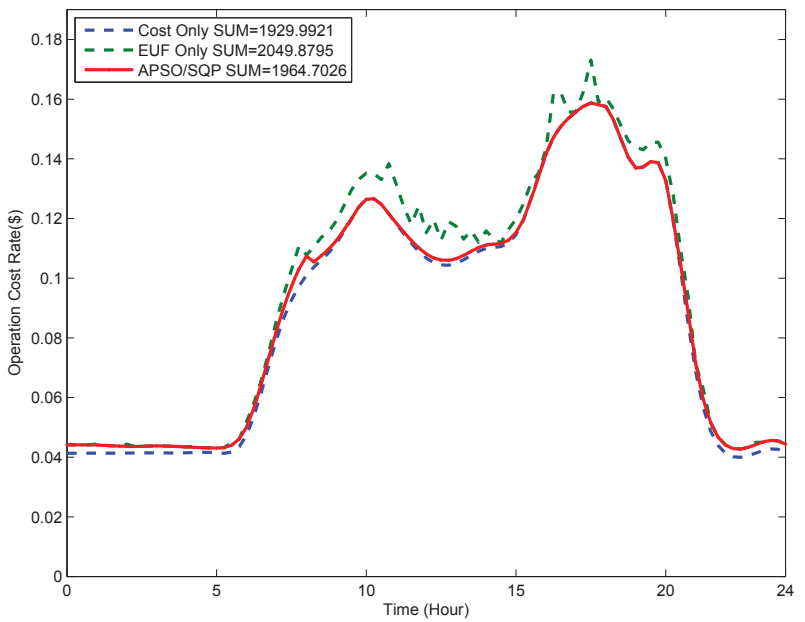

Fig. 6: Operational cost comparison in summer.

Figs. 7 and 8 show the results of EUF and operational cost in transition season, respectively. Similar to the results in summer, the optimized mode can achieve the highest efficiency at some point. As expected, the average efficiency of optimized mode case is between cost only case and EUF only case, but is a little lower than the CET case. It is also noted that the average system thermal efficiency in transition season is lower than those in summer and winter. This is probably due to the smaller thermal demand in transition season. In other words, more generated heat has to be wasted which leads to a lower system efficiency in transition season. For the operational cost, the result of optimized mode case is much closer to that of cost only case, and is always lower than the EUF only case.

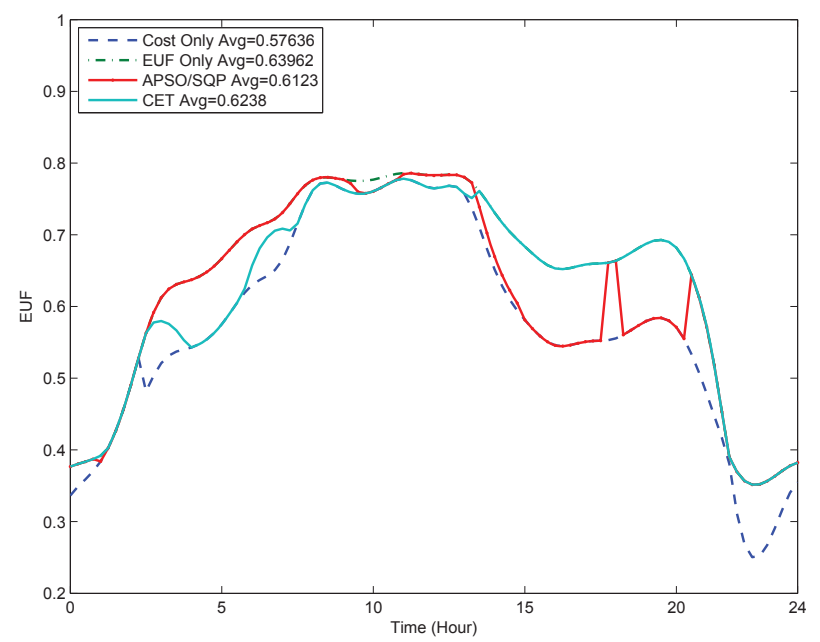

Fig. 7: EUF comparison in transition.

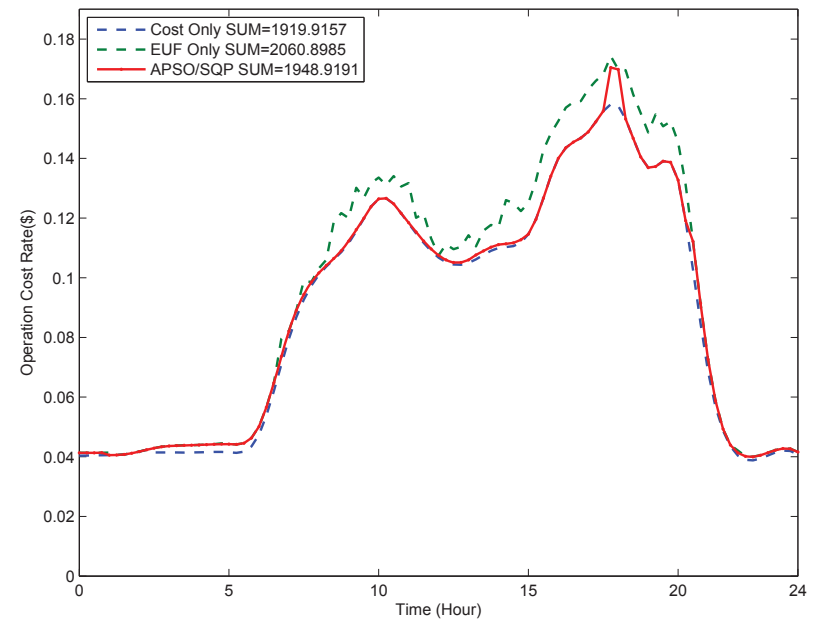

Fig. 8: Operational cost comparison in transition.

Figs. 9 and 10 show the efficiency and operational cost of four cases in winter. The efficiency of optimized mode case is found to be very close to the EUF only solution, and is higher than the CET case. For the operational cost, the trajectory of optimal operational cost coincides with the EUF only case at night when the thermal load is high and electrical load is relatively low. During the daytime, it is found that the optimized strategy operates closer to cost only curved than the efficiency only curve, suggesting that the proposed optimized strategy is capable of reducing the cost. The comparison of average operational cost among the three cases also confirms it. 


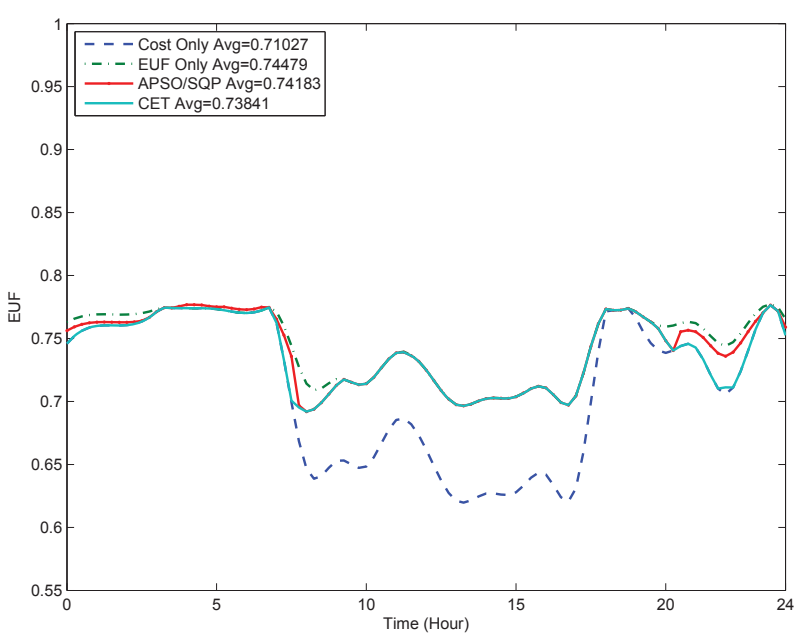

Fig. 9: EUF comparison in winter.

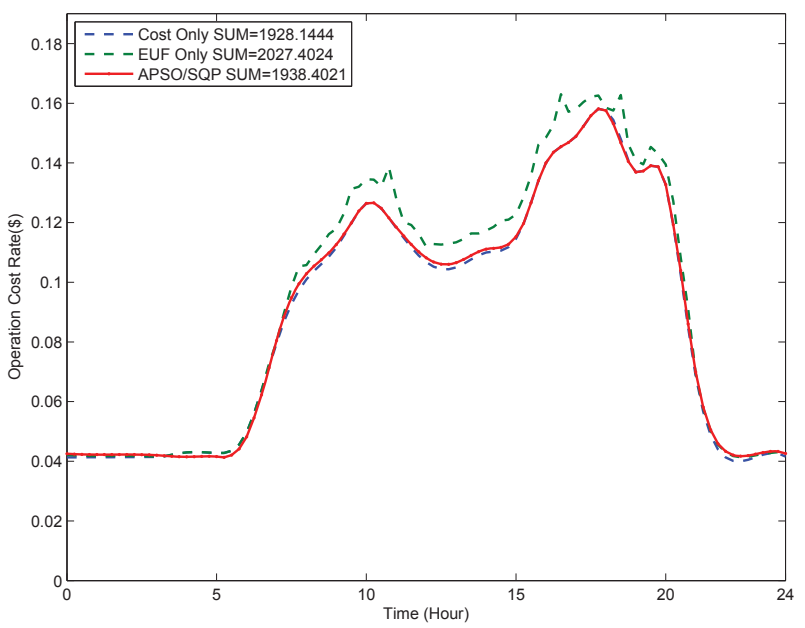

Fig. 10: Operational cost comparison in winter.

\section{CONCLUSION}

In this paper, a gas turbine-based DES has been developed using detailed component models. A multi-objective cost function including the system operation cost and the exergy efficiency has been proposed for obtaining optimal operating strategies. The optimization problem was formulated as a nonlinear programming problem that was solved by a two-stage approach combining PSO and SQP. Three cases under different demanding loads were studied using the proposed DES model. In comparison with the results obtained by rule-based methods, it is found that the optimized operation strategy using the twostage method can improve the system performance in terms of system operation cost and efficiency within an acceptable computational time. Therefore, it is believed that the two-stage method can be applied to DES planning and operation.

\section{REFERENCES}

[1] T. Ackermann, G. Andersson, and L. Söder, "Distributed generation: a definition," Electric power systems research, 57(3):195-204, 2001.
[2] R. Banos, F. Manzano-Agugliaro, and F.G. Montoya, "Optimization methods applied to renewable and sustainable energy: A review," Renewable and Sustainable Energy Reviews, 15(4):1753-1766, 2011.

[3] D. C. Walters and G. B. Sheble, "Genetic algorithm solution of economic dispatch with valve point loading," IEEE transactions on Power Systems, 8(3):1325-1332, 1993.

[4] G. Chicco and P. Mancarella, "Trigeneration primary energy saving evaluation for energy planning and policy development," Energy policy, 35(12):6132-6144, 2007.

[5] D. E. Santo, "Energy and exergy efficiency of a building internal combustion engine trigeneration system under two different operational strategies," Energy and Buildings, 53:28-38, 2012.

[6] J. Wang, C. Zhang, and Y. Jing, "Multi-criteria analysis of combined cooling, heating and power systems in different climate zones in china," Applied Energy, 87(4):1247-1259, 2010.

[7] N. K. Rizk and H. Mongia, "Semianalytical correlations for nox, co, and uhc emissions," In ASME 1992 International Gas Turbine and Aeroengine Congress and Exposition, pages V003T06A023-V003T06A023. American Society of Mechanical Engineers, 1992.

[8] P. Ahmadi, I. Dincer, and M. A. Rosen, "Exergo-environmental analysis of an integrated organic rankine cycle for trigeneration," Energy Conversion and Management, 64:447-453, 2012.

[9] W. Wang, R. Cai, and N. Zhang, "General characteristics of single shaft microturbine set at variable speed operation and its optimization," Applied thermal engineering, 24(13):1851-1863, 2004.

[10] N. Zhang and R. Cai, "Analytical solutions and typical characteristics of part-load performances of single shaft gas turbine and its cogeneration," Energy Conversion and Management, 43(9):1323-1337, 2002.

[11] S. P. Borg and N. J. Kelly, "The development and calibration of a generic dynamic absorption chiller model," Energy and Buildings, 55:533-544, 2012.

[12] A. L. Facci, L. Andreassi, and S. Ubertini, "Optimization of chcp (combined heat power and cooling) systems operation strategy using dynamic programming," Energy, 66:387-400, 2014.

[13] A. Rong, R. Lahdelma, and P. B. Luh, "Lagrangian relaxation based algorithm for trigeneration planning with storages," European Journal of Operational Research, 188(1):240-257, 2008.

[14] Z. Michalewicz and M. Schoenauer, "Evolutionary algorithms for constrained parameter optimization problems," Evolutionary computation, 4(1):1-32, 1996.

[15] L. Guo, W. Liu, J. Cai, B. Hong, and C. Wang, "A two-stage optimal planning and design method for combined cooling, heat and power microgrid system," Energy Conversion and Management, 74:433-445, 2013.

[16] R. Yokoyama, K. Ito, and Y. Matsumoto, "Optimal sizing of a gas turbine cogeneration plant in consideration of its operational strategy," Journal of engineering for gas turbines and power, 116(1):32-38, 1994.

[17] P. Mago, N. Fumo, and L. Chamra, "Performance analysis of cchp and chp systems operating following the thermal and electric load," International Journal of Energy Research, 33(9):852-864, 2009.

[18] Frank Incropera and David DeWitt. Introduction to heat transfer. 1985

[19] V. Chandan, A. Do, and B. Jin, "Modeling and optimization of a combined cooling, heating and power plant system," In American Control Conference (ACC), 2012, pages 3069-3074. IEEE.

[20] L. A. Vardhan and A. Vasan, "Evaluation of penalty function methods for constrained optimization using particle swarm optimization," In Image Information Processing (ICIIP), 2013 IEEE Second International Conference on, pages 487-492. IEEE, 2013. 